\title{
ON THE FOURIER DEVELOPMENTS OF A CERTAIN CLASS OF THETA QUOTIENTS
}

\author{
M. A. BASOCO
}

1. Introduction. In this paper we shall be concerned with the functions $\phi_{\alpha}^{k}(z)$ defined by the relation

$$
\phi_{\alpha}^{k}(z) \equiv\left\{\frac{d}{d z} \log \vartheta_{\alpha}(z, q)\right\}^{k}=\left\{\frac{\vartheta_{\alpha}^{\prime}(z, q)}{\vartheta_{\alpha}(z, q)}\right\}^{k}, \quad \alpha=0,1,2,3,
$$

where $\vartheta_{\alpha}(z, q)$ is a Jacobi theta function and $k$ is a positive integer. In the first place, we shall derive the Fourier developments which represent these functions in a certain strip of the complex plane; it will be seen that the Fourier coefficients of $\phi_{\alpha}^{k}(z)$ depend on those of $\phi_{\alpha}^{s}(z), s=1,2,3, \cdots, k-1$, through a recurrence relation of order $k$. Secondly, these developments, in conjunction with certain obvious identities, yield, through the method of paraphrase, some general arithmetical formulae of a type first given by Liouville. ${ }^{1}$ Indeed, we recover, in a simple manner, some results given without proof by Liouville, which were later proved by Bell ${ }^{2}$ through the use of somewhat complex identities involving a certain set of doubly periodic functions of the second kind. One of these results has recently been proved in a strictly elementary, but very ingenious way, by Uspensky. ${ }^{3}$ Finally, we indicate some applications of these formulae to the derivation of a certain type of arithmetic and algebraic identities.

2. The functions $\phi_{\alpha}^{k}(z)$. It should be pointed out that the case $k=1$, is implicit in $\$ \$ 47$ and 48 of Jacobi's Fundamenta nova. ${ }^{4}$ Likewise, the case $k=2$, has been obtained by G. D. Nichols ${ }^{5}$ through the use of certain results due to the present writer ${ }^{6}$ The following is a direct derivation of the necessary procedure for the general case; it depends on a straightforward application of contour integration and the theory of residues. It is convenient to treat the two functions $\phi_{0}^{k}(z)$ and 1942.

Presented to the Society, November 28, 1942; received by the editors June 29,

${ }^{1}$ J. Math. Pures Appl. (2) vol. 3 (1858) et seq. See, for example, vol. 3 p. 247 (H).

2 Trans. Amer. Math. Soc. vol. 22 (1921) p. 215 formula (xiv').

3 J. V. Uspensky and M. A. Heaslet, Elementary number theory, New York, 1939. See chap. 13 p. 462 formula (R).

${ }^{4} \mathrm{Jacobi}$, Gesammelte Werke, vol. 1 p. 187.

5 G. D. Nichols, Tôhoku Math. J. vol. 40 (1935) pp. 252-258.

6 M. A. Basoco, Bull. Amer. Math. Soc. vol. 38 (1932) pp. 560-568. 
$\phi_{1}^{k}(z)$ separately; from the results for these functions we may obtain the corresponding ones for $\alpha=2,3$ by merely replacing $z$ by $z+\pi / 2$.

It follows from the properties of the theta functions ${ }^{\bar{\tau}}$ and Fourier's theorem for analytic functions that

$$
\phi_{0}^{k}(z)=\sum_{n=-\infty}^{\infty} A_{n}^{(k)} e^{-2 n i z}, \quad-\Im(\pi \tau / 2)<\Im(z)<\Im(\pi \tau / 2),
$$

where,

$$
A_{n}^{(k)}=(1 / \pi) \int_{-\pi / 2}^{\pi / 2} \phi_{0}^{k}(z) e^{2 n i z} d z .
$$

To evaluate this integral, consider the contour integral $\int_{C} \phi_{0}^{k}(z) e^{2 n i z} d z$, the contour $C$ being the boundary of the parallelogram with vertices at $z= \pm \pi / 2$ and $z= \pm \pi / 2+\pi \tau / 2$. Cauchy's theorem applied to this integral leads without difficulty to the recurrence relation

$$
A_{n}^{(k)}-q^{2 n} \sum_{j=0}^{k-1} C_{k, j}(-2 i)^{j} A_{n}^{(k-j)}=2 i R, \quad q=\exp \pi i \tau,
$$

where $C_{k, j}$ is the binomial coefficient $k ! / j !(k-j) !$ and $R$ is the residue of the integrand at the pole of order $k$ with affix $z=\pi \tau / 2$.

We thus obtain, on placing $k=1,2,3$ and computing the necessary residues, the following expressions for the coefficients in the expansions of $\phi_{0}^{k}(z)$, for the values of $k$ indicated:

$$
\begin{aligned}
& \text { (a) } A_{0}^{(1)}=0 ; A_{n}^{(1)}=-A_{-n}^{(1)}=\frac{2 i q^{n}}{1-q^{2 n}}, \quad n \neq 0, \\
& \text { (b) } A_{n}^{(2)}=A_{-n}^{(2)}=\frac{8 q^{3}}{\left(1-q^{2 n}\right)^{2}}-\frac{4(n-1)}{1-q^{2 n}}, n \neq 0 ; A_{0}=-2 \sum_{n=1}^{\infty} A_{n}^{(2)} \\
& \text { (c) } A_{0}^{(3)}=0 ; A_{n}^{(3)}=-A_{-n}^{(3)}=\frac{\vartheta_{1}^{\prime \prime \prime}}{\vartheta_{1}^{\prime}} \cdot \frac{2 i q^{n}}{1-q^{2 n}} \\
&-\frac{2 i q^{n}}{\left(1-q^{2 n}\right)^{3}}\left\{\left(2 n^{2}+6 n+3\right) q^{4 n}\right. \\
&\left.\quad+\left(18-4 n^{2}\right) q^{2 n}+\left(2 n^{2}-6 n+3\right)\right\} .
\end{aligned}
$$

With respect to the function $\phi_{1}^{k}(z)$ the process used in the preceding must be modified slightly on account of the presence of a singularity (pole of order $k$ ) at the origin. Define the function $\psi_{1}^{(k)}(z)$ to be of the form

${ }^{7}$ See Whittaker and Watson, Modern analysis, chap. 21. 


$$
\psi_{1}^{(k)}(z) \equiv \phi_{1}^{k}(z)-T^{(k)}(z),
$$

where $T^{(k)}(z)$ is a suitable function of $\sin z$ and $\cos z$, having the period $\pi$ and such that the principal part of its Laurent expansion about the origin coincides with that of $\phi_{1}^{k}(z)$. The function $\psi_{1}^{(k)}(z)$ is, therefore, analytic along the real axis. It is found that, for the cases considered (that is, for $k=1,2,3$ ), the functions $T^{(k)}(z)$ are of the form:

$T^{(1)}(z)=\cot z ; T^{(2)}(z)=\cot ^{2} z ; T^{(3)}(z)=\left(1+\vartheta_{1}^{\prime} / \vartheta_{1}^{\prime}\right) \cot z+\cot ^{3} z$.

We may now write

$$
\begin{aligned}
& \psi_{1}^{(k)}(z)=\sum_{n=1}^{\infty} B_{n}^{(k)}\left(e^{2 n i z}-e^{-2 n i z}\right), \text { if } k \text { is odd } \\
& \psi_{1}^{(k)}(z)=B_{0}^{(k)}+\sum_{n=1}^{\infty} B_{n}^{(k)}\left(e^{2 n i z}+e^{-2 n i z}\right), \text { if } k \text { is even. }
\end{aligned}
$$

These expansions are valid in the strip defined by the inequalities $-\Im(\pi \tau)<\Im(z)<\Im(\pi \tau)$. In either case, we have,

$$
B_{n}^{(k)}=(1 / \pi) \int_{-\pi / 2}^{\pi / 2} \psi_{1}^{(k)}(z) e^{-2 n i z} d z, \quad n \neq 0 .
$$

The value of $B_{0}^{(k)}$ can be obtained from the relation

$$
B_{0}^{(k)}=-2 \sum_{n=1}^{\infty} B_{n}^{(k)}+\lim _{z \rightarrow 0} \psi_{1}^{(k)}(z) .
$$

To calculate the integral in (6), consider the contour integral $\int_{C} \psi_{1}^{(k)}(z) e^{-2 n i z} d z$ where $C$ is the boundary of the parallelogram with vertices at $z= \pm \pi / 2$ and $z= \pm \pi / 2-(2 s+1) \pi \tau / 2$, where $s$ is an arbitrary positive integer. This contour contains precisely $s$ poles of the function $\psi_{1}^{(k)}(z)$, these being located at the points with affixes $z=-r \pi \tau, r=1,2,3, \cdots, s$. On account of the properties of the functions involved we may pass to the limit as $s$ tends to infinity, thus obtaining

$$
B_{n}^{(k)}=-2 i \sum_{s=1}^{\infty} R_{s}
$$

where $R_{s}$ is the residue of $\psi !^{(k)}(z) e^{-2 n i z}$ at the poles of order $k$, $z=-s \pi \tau$.

It is found in this manner that

$$
B_{0}^{(1)}=0 ; B_{n}^{(1)}=\frac{-2 i q^{2 n}}{1-q^{2 n}}, \quad n \neq 0 .
$$


(e) $B_{0}^{(2)}=8 \sum_{n=1}^{\infty} \frac{n q^{2 n}}{1-q^{2 n}} ; B_{n}^{(2)}=\frac{8 q^{2 n}}{\left(1-q^{2 n}\right)^{2}}-\frac{4 n q^{2 n}}{1-q^{2 n}}, \quad n \neq 0$.

(f) $\quad B_{0}^{(3)}=0 ; B_{n}^{(3)}=\frac{\vartheta_{1}^{\prime \prime \prime}}{\vartheta_{1}^{\prime}} \cdot \frac{4 q^{2 n}}{1-q^{2 n}}$

$$
\begin{array}{r}
-\frac{8 q^{2 n}}{\left(1-q^{2 n}\right)^{3}}\left\{n^{2} q^{4 n}-\left(2 n^{2}-6 n-6\right) q^{2 n}+\left(n^{2}-6 n+6\right)\right\} \\
n \neq 0 .
\end{array}
$$

On substituting the values of the coefficients given in (a), . , (f), into (2) and (5) and transforming the resulting series into an arithmetical form, we find the following:

(I) $\quad \phi_{0}(z)=4 \sum_{(n)} q^{n}\left\{\sum \sin 2 t z\right\}, \quad n=1,2,3,4, \cdots$,

(II) $\quad \phi_{1}(z)=\cot z+4 \sum_{(n)} q^{2 n}\left\{\sum \sin 2 d z\right\}$,

(III) $\quad \phi_{2}(z)=-\tan z+4 \sum_{(n)} q^{2 n}\left\{\sum(-1)^{d} \sin 2 d z\right\}$,

(IV) $\quad \phi_{3}(z)=4 \sum_{(n)}(-1)^{n} q^{n}\left\{\sum \sin 2 t z\right\}$

(V) $\quad \phi_{0}^{2}(z)=8 \sum_{(n)} q^{n} \sigma(n)+8 \sum_{(n)} q^{n}\left\{\sum(\tau-t) \cos 2 t z\right\}$,

(VI) $\quad \phi_{1}^{2}(z)=\cot ^{2} z+8 \sum_{(n)} q^{2 n} \zeta_{1}(n)+8 \sum_{(n)} q^{2 n}\left\{\sum(2 \delta-d) \cos 2 d z\right\}$, $\phi_{2}^{2}(z)=\tan ^{2} z+8 \sum_{(n)} q^{2 n} \zeta_{1}(n)$

$$
-8 \sum_{(n)} q^{2 n}\left\{\sum(-1)^{d}(2 \delta-d) \cos 2 d z\right\}
$$

(VIII) $\phi_{3}^{2}(z)=8 \sum_{(n)}(-1)^{n} q^{n} \sigma(n)+8 \sum_{(n)}(-1)^{n} q^{n}\left\{\sum(\tau-t) \cos 2 t z\right\}$,

(IX) $\quad \phi_{0}^{3}(z)=\left(\vartheta_{1}^{\prime \prime \prime} / \vartheta_{1}^{\prime}\right) \phi_{0}(z)-4 \sum_{(n)} q^{n}\left\{\sum\left(3 \tau^{2}-6 t \tau+2 t^{2}\right) \sin 2 t z\right\}$,

$$
\phi_{1}^{3}(z)=\cot z+\cot ^{3} z+\left(\vartheta_{1}^{\prime \prime \prime} / \vartheta_{1}^{\prime}\right) \phi_{1}(z)
$$

$$
-8 \sum_{(n)} q^{2 n}\left\{\sum\left(d^{2}-6 d \delta+6 \delta^{2}\right) \sin 2 d z\right\},
$$

$$
\phi_{2}^{3}(z)=-\tan z-\tan ^{3} z+\left(\vartheta_{1}^{\prime \prime \prime} / \vartheta_{1}^{\prime}\right) \phi_{2}(z)
$$

$$
-8 \sum_{(n)} q^{2 n}\left\{\sum(-1)^{d}\left(d^{2}-6 d \delta+6 \delta^{2}\right) \sin 2 d z\right\},
$$




$$
\begin{aligned}
& \phi_{3}^{3}(z)=\left(\vartheta_{1}^{\prime \prime} / \vartheta_{1}^{\prime}\right) \phi_{3}(z) \\
&-4 \sum_{(n)}(-1)^{n} q^{n}\left\{\sum\left(3 \tau^{2}-6 t \tau+2 t^{2}\right) \sin 2 t z\right\}, \\
&\left(\vartheta_{1}^{\prime \prime \prime} / \vartheta_{1}^{\prime}\right)=-1+24 \sum_{(n)} q^{2 n} \zeta_{1}(n) .
\end{aligned}
$$

In these expansions, the notation is as usual: The inner sigma refers to the divisors $d, \delta, t, \tau$ of the positive integer $n, \tau$ being odd, and

where,

$$
\sigma(n)=\psi_{1}(n)-\zeta_{1}^{\prime}(n)
$$

$\psi_{1}(n)=$ sum of the divisors of $n$ whose conjugates are odd,

$\zeta_{1}^{\prime}(n)=$ sum of the odd divisors of $n$, and

$\zeta_{1}(n)=$ sum of all the divisors of $n$.

3. Paraphrases. The obvious identities

$$
\phi_{a}(z) \cdot \phi_{a}(z)=\phi_{a}^{2}(z), \quad a=0,1,2,3,
$$

in conjunction with the expansions (I) to (VIII) of the preceding section yield, through the method of paraphrase, ${ }^{8}$ the following arithmetical theorems:

THEOREM $(\alpha)$. Let $F(x)$ be an even, single-valued function which is well defined for all integral values of the argument including zero, but is otherwise arbitrary; let $n$ be any positive integer. Then,

$$
\sum_{(k)}\left\{F\left(t^{\prime}-t^{\prime \prime}\right)-F\left(t^{\prime}+t^{\prime \prime}\right)\right\}=\sum_{(l)}(\tau-t)\{F(t)-F(0)\},
$$

where (k) and (l) refer to the following partitions of $n$ in positive integers:

$$
\text { (k) } n=t^{\prime} \tau^{\prime}+t^{\prime \prime} \tau^{\prime \prime}, \quad(l) \quad n=t \tau, \quad \tau, \tau^{\prime}, \tau^{\prime \prime} \text { odd. }
$$

This is the arithmetical equivalent of (8) with $a=0$. It is of interest to note that a strictly elementary proof of this theorem has recently been given by Uspensky. ${ }^{3}$

THEOREM $(\beta)$. Let $F(x)$ and the integer $n$ be as in Theorem $(\alpha)$. Then,

$$
\begin{aligned}
\sum_{(i)}\left\{F\left(d^{\prime}-d^{\prime \prime}\right)-\right. & \left.F\left(d^{\prime}+d^{\prime \prime}\right)\right\} \\
= & \left\{\zeta_{1}(n)-\zeta_{0}(n)\right\} F(0)+\sum_{(j)}(2 \delta-d-1) F(d) \\
& -2 \sum_{(j)}\{F(1)+F(2)+F(3)+\cdots+F(d-1)\}
\end{aligned}
$$

${ }^{8}$ E. T. Bell, Trans. Amer. Math. Soc. vol. 22 (1921) pp. 1-30; 198-219. 
where $(i),(j)$ refer to the following partitions of $n$ in positive integers:

$$
\text { (i) } n=d^{\prime} \delta^{\prime}+d^{\prime \prime} \delta^{\prime \prime}, \quad(j) \quad n=d \delta .
$$

$\zeta_{0}(n)$ represents the number of positive integral divisors of $n$, while $\zeta_{1}(n)$ represents their sum.

This theorem was first given by Liouville, ${ }^{9}$ who stated it without giving a proof; it is conjectured that he obtained this as well as other similar results through the aid of the theory of elliptic functions. At any rate, this theorem was later rediscovered by Bell (loc. cit.) as a special case of a more general result derived from one of the addition theorems of the theta functions and involving a certain set of doubly periodic functions of the second kind. We have obtained this result as the equivalent of the identity (8) with $a=1$.

ThEOREM $(\gamma)$. Let $F(x), n$, and the partitions $(i)$ and $(j)$ be as in the preceding theorem. Then,

$$
\begin{aligned}
\sum_{(i)}(-1)^{d^{\prime}+d^{\prime \prime}} & \left\{F\left(d^{\prime}-d^{\prime \prime}\right)-F\left(d^{\prime}+d^{\prime \prime}\right)\right\} \\
= & \left\{\zeta_{1}(n)-\zeta_{0}(n)\right\} F(0)-\sum_{(j)}(-1)^{d}(2 \delta-d+1) F(d) \\
& +2 \sum_{(j)}\left\{F(1)-F(2)+F(3)-\cdots+(-1)^{d} F(d-1)\right\} .
\end{aligned}
$$

This theorem is the arithmetical equivalent of identity (8) with $a=2$. It is, however, not essentially distinct from Theorem $(\beta)$; for either theorem may be obtained from the other by replacing $F(x)$ by $(-1)^{x} F(x)$. Finally it should be noted that the case $a=3$ merely yields the result stated in Theorem $(\alpha)$.

As might be expected, those theorems analogous to the preceding which may be deduced from identities involving the functions $\phi_{a}^{3}(z)$ are considerably more complicated; it will perhaps suffice, in the present instance, to state merely the following two results by way of samples.

THEOREM ( $\delta)$. Let $G(x)$ be an odd, single-valued function, which is well defined for all integral values of the argument, but is otherwise arbitrary; let $n$ be any positive integer. Then,

$$
\begin{array}{r}
4 \sum_{(i)}\left\{G\left(t_{1}-t_{2}+t_{3}\right)+G\left(t_{1}+t_{2}-t_{3}\right)+G\left(t_{2}+t_{3}-t_{1}\right)-G\left(t_{1}+t_{2}+t_{3}\right)\right\} \\
=24 \sum_{(j)}\left\{\zeta_{1}\left(n_{1}\right) \sum G\left(t_{2}\right)\right\}-\sum_{(k)}\left(3 \tau^{2}-6 t \tau+2 t^{2}+1\right) G(t),
\end{array}
$$

${ }^{9}$ Liouville, loc. cit. 
where ( $i),(j)$ and $(k)$ refer to the following partitions of $n$ in positive integers:

$$
\begin{array}{rlr}
\text { (i) } \quad n=t_{1} \tau_{1}+t_{2} \tau_{2}+t_{3} \tau_{3} & \tau_{1}, \tau_{2}, \tau_{3} \text { all odd, } \\
\text { (j) } \quad n=2 n_{1}+n_{2}, \quad n_{2}=t_{2} \tau_{2}, & \tau_{2} \text { odd, } \\
\text { (k) } n & n=t \tau, & \tau \text { odd. }
\end{array}
$$

This theorem is the arithmetical equivalent of the otherwise apparently trivial identity:

$$
\phi_{0}(z) \cdot \phi_{0}(z) \cdot \phi_{0}(z) \equiv \phi_{0}^{3}(z) .
$$

Theorem ( $\epsilon)$. Let $F(x), n$, and the partitions $(i)$ and $(j)$ be as in Theorem $(\beta)$. Then,

$$
\begin{aligned}
6 \sum_{(i)} d^{\prime}\left(d^{\prime \prime}-\right. & \left.2 \delta^{\prime \prime}\right)\left\{F\left(d^{\prime}+d^{\prime \prime}\right)+F\left(d^{\prime}-d^{\prime \prime}\right)\right\} \\
= & 6\left(n \zeta_{0}(n)-\zeta_{2}(n)\right) F(0)+12 \sum_{j}(\delta-d)\left\{\sum_{r=1}^{d} \sum_{\delta=1}^{r-1} F(s)\right\} \\
& +\sum_{(j)}\left(d^{3}-6 d^{2} \delta+6 d \delta^{2}-d\right) F(d) .
\end{aligned}
$$

This theorem results on paraphrasing the identity:

$$
\frac{d}{d z} \phi_{1}^{3}(z) \equiv 3 \phi_{1}^{2}(z) \frac{d}{d z} \phi_{1}(z)
$$

4. An application. If in Theorem $(\alpha)$ we place $F(x)=x^{2}$, the following may be deduced

$$
4 \sum_{k=1}^{n-1} \psi_{1}(k) \psi_{1}(n-k)=\psi_{3}(n)-n \psi_{1}(n),
$$

where $\psi_{r}(n)=$ sum of the $r$ th powers of the divisors of $n$ whose conjugates are odd. In particular, if $n$ is a prime $p$, we obtain

$$
4 \sum_{k=1}^{p-1} \psi_{1}(k) \psi_{1}(p-k)=(p-1)\left(p^{2}-1\right) .
$$

It also follows from (9) and the easily verified relationships:

$$
\begin{gathered}
\sum_{n=1}^{\infty} \frac{n x^{n}}{1-x^{2 n}}=\sum_{n=1}^{\infty} \psi_{1}(n) x^{n}, \quad \sum_{n=1}^{\infty} \frac{n^{3} x^{n}}{1-x^{2 n}}=\sum_{n=1}^{\infty} \psi_{3}(n) x^{n}, \\
\sum_{n=1}^{\infty} \frac{n^{2}\left(1+x^{2 n}\right) x^{n}}{\left(1-x^{2 n}\right)^{2}}=\sum_{n=1}^{\infty} n \psi_{1}(n) x^{n},
\end{gathered}
$$


that,

$$
\begin{gathered}
4\left\{\sum_{n=1}^{\infty} \frac{n x^{n}}{1-x^{2 n}}\right\}^{2}=\sum_{n=1}^{\infty} \frac{n^{3} x^{n}}{1-x^{2 n}}-\sum_{n=1}^{\infty} \frac{n^{2} x^{n}}{\left(1-x^{2 n}\right)^{2}} \\
-\sum_{n=1}^{\infty} \frac{n^{2} x^{3 n}}{\left(1-x^{2 n}\right)^{2}} .
\end{gathered}
$$

Again, if in Theorem $(\beta)$ we place $F(x)=x^{2}$, we deduce

$$
12 \sum_{k=1}^{n-1} \zeta_{1}(k) \zeta_{1}(n-k)=5 \zeta_{3}(n)-(6 n-1) \zeta_{1}(n),
$$

which in turn implies the following development,

$$
12\left\{\sum_{n=1}^{\infty} \frac{n x^{n}}{1-x^{n}}\right\}^{2}=\sum_{n=1}^{\infty}\left(5 \zeta_{3}(n)-(6 n-1) \zeta_{1}(n)\right) x^{n} .
$$

Finally, in Theorem $(\delta)$ place $G(x)=x$ to deduce

$$
\begin{aligned}
& 24 \sum \zeta_{1}\left(n_{1}\right) \psi_{1}\left(n_{2}\right)=2 \psi_{3}(n)-(6 n-1) \psi_{1}(n)+3 n \zeta_{1}^{\prime}(n), \\
& n=2 n_{1}+n_{2}, n_{1}, n_{2}>0 .
\end{aligned}
$$

This result may be interpreted so as to yield the following relation:

$$
\begin{gathered}
24\left\{\sum_{n=1}^{\infty} \frac{n x^{2 n}}{1-x^{2 n}}\right\} \cdot\left\{\sum_{n=1}^{\infty} \frac{n x^{n}}{1-x^{2 n}}\right\} \\
=2 \sum_{n=1}^{\infty} \frac{n^{3} x^{n}}{1-x^{2 n}}+\sum_{n=1}^{\infty} \frac{n x^{n}}{1-x^{2 n}}-6 \sum_{n=1}^{\infty} \frac{n^{2}\left(1+x^{2 n}\right) x^{n}}{\left(1-x^{2 n}\right)^{2}} \\
+3 \sum_{n=1}^{\infty} \frac{(2 n-1)^{2} x^{2 n-1}}{\left(1-x^{2 n-1}\right)^{2}} .
\end{gathered}
$$

Results analogous to the preceding, but developed from an entirely different point of view, have been obtained by Glaisher. ${ }^{10}$ Here, such results appear as very special consequences of the general theorems stated in \$3. It is clear, of course, that similar results may be obtained through the full use of the expansions here given.

The UNIVERSITY OF NeBRASKa

${ }^{10} \mathrm{~J}$. W. L. Glaisher, Messenger of Mathematics nos. 166, 169 (1885). 